\title{
Towards QoS Provisioning in a Heterogeneous Carrier-Grade Wireless Mesh Access Networks Using Unidirectional Overlay Cells
}

\author{
M. Kretschmer ${ }^{1}$, C. Niephaus ${ }^{1}$, and G. Ghinea ${ }^{2}$ \\ 1 Fraunhofer FOKUS, Sankt Augustin, Germany \\ \{mathias.kretschmer, christian.niephaus\}@fokus.fraunhofer.de \\ 2 Brunel University, London, England \\ george.ghinea@brunel.ac.uk
}

\begin{abstract}
The visibility and success of Wireless Mesh Network (WMN) deployments has raised interest among commercial operators in this technology. Compared to traditional operator access networks WMNs have the potential to offer easier deployment and flexible self-reconfiguration at lower costs. A WMN-type architecture considered as an alternative for an operator access network must meet similar requirements such as high availability and guaranteed QoS in order to support triple-play content provisioning. In this paper we introduce an architecture of such a Carrier-grade Wireless Mesh Access Network (CG-WMAN). We then present our contribution, an approach to seamlessly integrate unidirectional broadcast cells (i.e. DVB-T) into such a CG-WMAN. This allows higher layer protocols to utilize broadcast cells like regular mesh links, where beneficial for a given payload and receiver distribution. We then present a typical use case and discuss for which combinations of traffic type, user distribution and QoS requirements the use of longer range broadcast technologies can help to improve the overall CG-WMAN performance in terms of throughput and reliability.
\end{abstract}

\section{Introduction}

WMNs have attracted the attention of network operators due to their increased deployment flexibility and potentially lower operational costs compared to regular rather fixed wireless operator networks. The work presented in this paper has been done within the context of the CARrier grade wireless MEsh Network (CARMEN) 2] project, which aims at studying and specifying a WMN supporting carrier grade triple-play services in future heterogeneous mobile/fixed network operator environments. A CARMEN access network can complement existing access technologies by exploiting low costs mesh networking techniques. A key component of this CG-WMAN is an abstraction layer based on and extending IEEE 802.21 to allow the integration of heterogeneous wireless technologies such as IEEE 802.11, IEEE 802.16 as well as Digital Video Broadcast (DVB) and 3rd Generation Partnership Project (3GPP) technologies) in a multi-hop fashion in order to provide ubiquitous Internet access in a scalable and efficient 
manner. On the control plane, the abstraction layer maps technology specific primitives onto a common set of events and commands. Upper layer modules such as self-configuration, routing, mobility management and monitoring are implemented on top of those abstract primitives and can therefore operate with any technology that provides a proper MAC adaptor. The concept of Traffic Engineering (TE) using Path Computation Elements (PCEs) as specified in [4] is adapted by the routing module to perform inter and intra area routing. Our work focuses of the seamless integration of unidirectional technologies so that they can be utilized when beneficial for a specific content or user distribution.

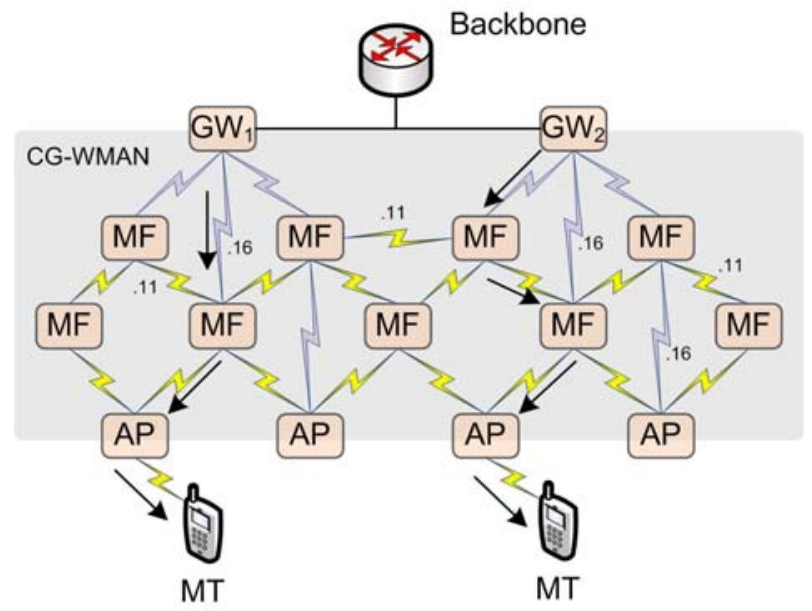

Fig. 1. Typical CG-WMAN scenario

Figure 1depicts a typical CG-WMAN scenario with dedicated gateways, mesh forwarder as well as access point nodes and various links interconnecting them. The links might have been establish using heterogeneous technologies such as IEEE 802.11 or 802.16 , chosen to optimally fit the deployment scenario with regards to financial constraints, range, spectrum availability and robustness.

Delivering triple-play services within a CG-WMAN is a challenging task since the delivery of high-bandwidth multimedia traffic substantially increases the load on the affected individual mesh links, the link groups or broadcast domains they belong to and therefore the CG-WMAN as a whole. We therefore propose the seamless integration of broadcast technologies as an efficient delivery medium especially for, but not limited to, broadcast and multicast content.

Figure 2 depicts the architecture of a CG-WMAN node. The central component of the control plane is the Media Independent Messaging Function (MIMF) which can be seen as an extension of the IEEE 802.21 Media Independent Handover Function (MIHF) providing additional mesh network related primitives. It also implements a module to module communication mechanisms. Additional IEEE 802.21 compliant message types have been defined to cover this extended 
Control Plane

\section{Data Plane}

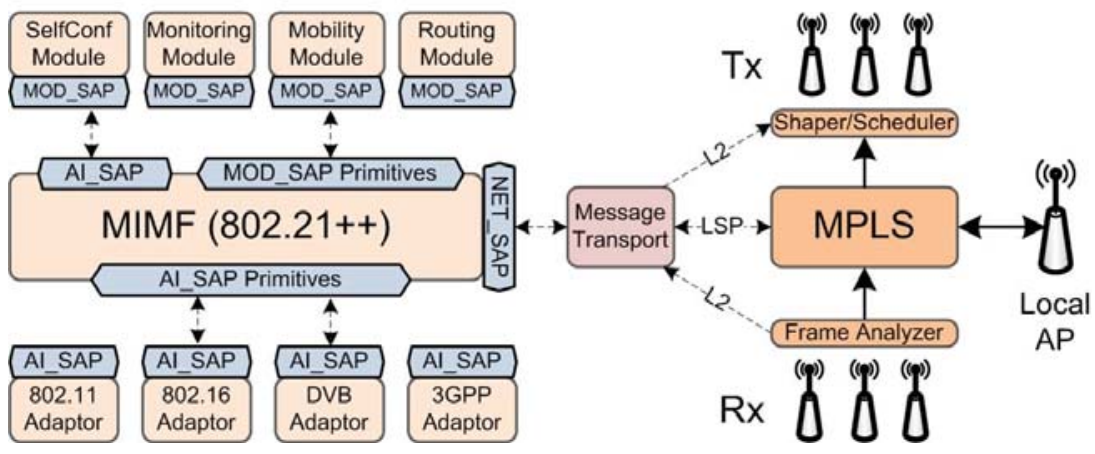

Fig. 2. The CG-WMAN Architecture is based on $802.21++$ and MPLS

functionality. Messages are forwarded within the CG-WMAN using either a dedicated management Multi Protocol Label Switching (MPLS) Label-Switched Paths (LSPs) or via hop-by-hop data link layer forwarding. The latter can choose between controlled flooding or explicit source routing. The CG-WMAN internally uses EUI-64 addresses, to allow for the incorporation of non-IEEE 802 technologies. Depending on the capabilities of the technology used, the wireless interfaces are operated in a promiscuous mode so that all frames received by the wireless network adapter can be analyzed by the Frame Analyzer component. This component is crucial to the proper operation of the CG-WMAN since it extracts and derives information from the received frames and their headers in order to allow the monitoring component to provide this information in a standardized manner to the other CG-WMAN modules.

Logically below the MIMF component, the MAC adaptors are located. They provide the adaptation of technology specific features to the common set of primitives provided by MIMF to the upper layer modules via the AI_SAP.

The higher layer modules combined provide the functionality of a traditional routing protocol - and beyond. The functionality of a module varies depending on the function of a node. Our CG-WMAN design is based on a centralized approach, where the centralized management nodes maintain the state of their area following the concept of a centralized and stateful PCE[4]. In the centralized approach unicast or multicast path computation as well as resource accounting and allocation can be performed optimally according to TE policies set forth by the operator. Due to the centralized management, it is crucial for the proper operation of the CG-WMAN that the state maintained at the management entity closely reflects the actual state of the physical mesh links.

The forwarding component is designed based on the MPLS [17] specification and will be described in more detail in the next section. The so-called link group concept has been introduced to address the issue of wireless channel resources being shared by more than one transmitting node. This concept takes into account medium access protocol characteristics, the resources allocated as well as 
the modulation being used in order to accurately compute and distribute the channel resources thus avoiding overbooking or contention. For infrastructurebased technologies such as IEEE 802.16 or 802.11 in managed mode, the nodes connected to the base station form a link group with the base station being the link group leader. In the case of a multicast LSP with multiple receivers in a link group, the datagrams need to be sent and accounted for with the smallest common modulation between the sending or relaying node, which is usually the base station, and the set of receivers.

The self configuration component is responsible for proper configuration of the mesh nodes, in particular it performs on-line radio planning to configure the wireless interfaces minimizing the possibility of interferences and maximizing the overall mesh network throughput. The main task is located at the management entity where it maintains a table of all possible physical links between nodes and their radios. A subset of those physical links is exported as a table of logical links which can be used for actual datagram forwarding. Physical and logical links are described as unidirectional resources. This table of logical links is similar to a link state table of traditional routing protocols such as Open Shortest Path First (OSPF) 12] and forms the basis of the TE path computation function. The details of self configuration module are out of the scope of this paper.

The mobility component provides Mobile Terminal (MT) mobility similar to the Proxy Mobile IP (PMIP) [5] concept, but is outside of the scope of this work.

\subsection{Overlay Cells}

Overlay cells have been studied in the literature for cellular networks where they might increase the system capacity [19] 8] 3], but also in the context of WMNs [16] 20] 11] [6]. Here mostly with the focus to break with the single-radioper-node ad-hoc forwarding paradigm and its limitations regarding throughput and predictable Quality of Service (QoS) support. In our CG-WMAN, bidirectional overlay cells are natively supported by our architecture since to our routing module they simply appear as longer distance links between mesh nodes in the link-state table, see Figure 1. The advantage of such links, the direct link local connection between nodes needs to be balanced against the lower bandwidth per area density compared to smaller mesh cells which can exploit Space-division multiple access (SDMA) and frequency re-use, see Figure 3. Smaller cells allow for higher unicast throughput via multiple hops, while larger cells can reach a large group of receivers with a single isochronous transmission. They are therefore well suited for the distribution of multicast traffic or specific mesh network management or synchronization tasks. Hence, we propose the use of overlay cells provided via robust unidirectional broadcast technologies such as Digital Video Broadcast - Terrestrial (DVB-T), which allow for one single sending node only. Hence, complicated Media Access Control (MAC) protocols can be omitted, thus freeing up more wireless channel resources for the actual data transmission, which yields a higher physical channel utilization efficiency.

Most multicast use cases can be addressed using 1-to-N trees. Where multiple or mobile senders are required, they could be configured to send their datagrams 
via unicast to the multicast tree root, which would then reflect them back out into the tree. This approach may increase the delay for some receiving nodes, but can easily be integrated into the CG-WMAN QoS management, mobility and forwarding schemes.

\section{$\mathrm{C}_{1} \mathrm{C}_{2} \mathrm{C}_{1}$ \\ $\mathrm{C}_{3} \mathrm{C}_{4} \mathrm{C}_{3}$ \\ $\mathrm{C}_{2} \mathrm{C}_{1} \mathrm{C}_{2}$ \\ overlay}

Fig. 3. Overlay cells have a higher range, but less dedicated bandwidth density

The CG-WMAN's routing module must utilize proper routing metrics to match a path resource request with given QoS requirements to the best matching links taking into account hop-count, bandwidth, costs and modulation constraints to reach all receivers.

In a CG-WMAN unidirectional overlay cells might only cover parts of the mesh, therefore further in-mesh multicast forwarding might be needed to reach all receivers, see Figure 4. We therefore propose to seamlessly integrate unidirectional overlay cells into the CG-WMAN architecture, so that they are seen by upper layer management modules such as routing like any other technology. Then, the routing module can automatically chose between overlay cells and regular mesh links or consider regular mesh links as extension branches of the overlay cell when computing an optimal multicast tree. If supported by the underlaying technology, the management modules could adapt the transmit power to control the cell size or balance between higher modulation or longer range.

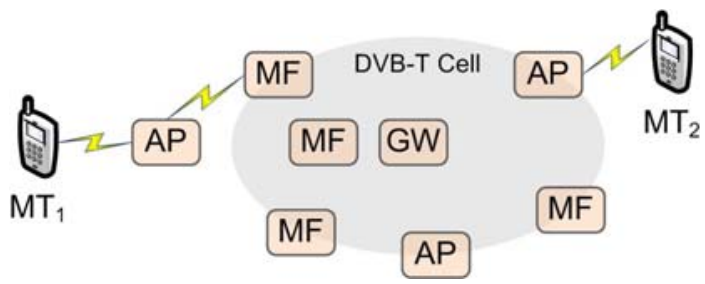

Fig. 4. A Unidirectional DVB-T overlay cell partially covering the CG-WMAN

As discussed in [10], the seamless integration of unidirectional technologies has implications on several CG-WMAN protocols and components, which usually 
expect bidirectional links between nodes. In the following section we will address those issues and present our proposed solutions. In section three, we will analyze a typical overlay cell use case. In section four we summarize our contribution and give an outlook on ongoing and future work.

\section{Approach: Integration of Unidirectional Technologies}

The CG-WMAN described in the previous section adopted many proven TE concepts and protocols which are in use today in MPLS-based carrier backbone networks. Such networks are built on top of reliable bidirectional links and run Interior Gateway Protocols (IGPs) such as OSPF 12] or Intermediate system to intermediate system (IS-IS) [9]. In this section we describe the adaptations that are required in order to natively support Unidirectional Links (UDLs) in a more volatile MPLS-based CG-WMAN.

As discussed in [10] our CG-WMAN uses a fully centralized and stateful QoSconstrained path computation scheme based on the Dijkstra algorithm which natively considers UDLs if present in the link state table. Path computation can be performed for unicast paths as well as for multicast trees. Forwarding is performed along MPLS LSPs which are unidirectional resources and, once configured, do not require any modification.

As described in the previous section, our CG-WMAN does not run a link state IGP, mainly due to their convergence issues in the presence of volatile links. It rather relies on a centralized management entity. As a consequence, network management protocols can not assume a functional Internet Protocol (IP) routing between mesh nodes. Additionally, when considering UDLs, protocols like Address Resolution Protocol (ARP) or Internet Protocol, Version 6 (IPv6) Stateless Address Autoconfiguration (SAA) can not be relied on. Hence, local scope IP addresses would need to be configured via a specifically tailored protocol. Without an operational routing protocol, though, the use of IP as the signaling layer does not provide any benefit. It rather introduces extra overhead due to the additional Internet Protocol (IPv4) or IPv6 header. Avoiding this overhead and addressing unidirectional MAC layer implementation, we propose to perform CG-WMAN messaging on the date link layer. In the centralized approach, control communication happens mainly between the management entity, which may be co-located with a gateway node, and a regular mesh node. If the management entity is not located at a gateway node, a proxy is required.

\section{$2.1 \quad$ MPLS}

The forwarding function supports multicast forwarding via a list of possible outputs, node-local scope labels as well as Fast Reroute (FRR) 13] to support fast fail-overs [15] in the case of link degradations below a pre-configured threshold. The forwarding component is designed based on the MPLS specification, but has been adapted to take into account UDLs as well as possible label collisions due to shared wireless channels where multiple sending nodes within a broadcast 
domain or link group might be upstream neighbors which independently assign the same label. An upstream label negotiation, as described in RFC3031 is not possible in the presence UDLs. To address this issue, the forwarding component switches based on so-called Point-to-Multipoint Labels (PMPLabels) on the incoming side which consist of the EUI64 address of the sending interface and the actual MPLS label, effectively turning the PMPLabel into a unique 84bit label. Due to the larger label size, a label can no longer be used as an index into a table. But, even a regular 20bit label would require a table with 1048576 entries, which might not fit into the memory of a small footprint mesh node. Hence, instead our forwarding module uses a simple hash function and stores its LSP state in a hash map. An alternative solution would be a centralized label assignment at the management entity. This approach has not been followed since it would increase the complexity of the management function even more.

While the setup and tear down protocol is related to Resource ReSerVation Protocol - Traffic Engineering (RSVP-TE), it does not need to allocate link resources, since this is handled centrally at the management entity. It might however carry additional information about the LSP's QoS requirements so that each node can configure it's traffic shapers or MAC schedulers accordingly. This information also has to be cleared on a tear down.

Regarding FRR, in the current IEEE 802.21 compatible design, a Point of Local Repair (PLR) would subscribe to LSP_REROUTE events of all downstream nodes that are allowed to trigger an FRR action. This could lead to a storm of events since all nodes downstream of a possible link breakage will detect and signal this event at almost the same time. To address this issue, a more efficient, probably hierarchical, delivery mechanisms suppressing identical messages from downstream nodes should be investigated.

\subsection{Monitoring}

Monitoring is the core component of a CG-WMAN since it performs radio as well as frame analysis of every frame received on any of a node's interfaces. This raw information is then categorized and interpreted by one or more of the monitoring levels, namely the radio level, the link level and the flow or LSP level, see Figure 5. Information processing can be done node local or at a centralized entity which can also correlate data in order to, for example, locate sources of interference.

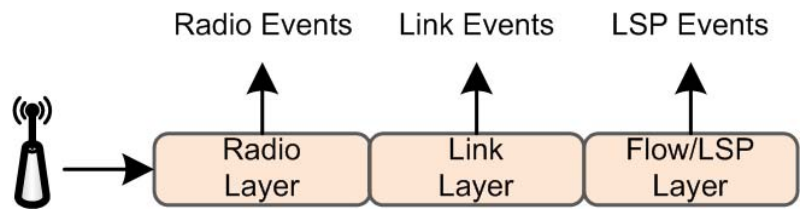

Fig. 5. The monitoring component evaluates samples at different layers 
The monitoring component can create a set of events when the state of the monitored object has changed. These events trigger most actions of the other CG-WMAN management components, such as:

- Change of Neighboring mesh node statistics

- Mobile Terminal (MT) presence detection and hand-over indications

- Indication of interference or intrusion

- Change of link state, i.e. up, down or underperforming

- Provision of LSP end-to-end performance statistics

- Creation of QoS \& network state aware LSP events to support i.e. FRR

- Network reconfiguration based on long term link analysis

Detailed wireless monitoring is a difficult task due to the dynamic nature of wireless links caused by temporary fading and interferences, but also due to often very dynamic per-frame transmitter configurations. The wireless technologies considered in our CG-WMAN range from satellite (i.e. DVB-S) over 3GPP to IEEE 802.16 and 802.11 . Therefore the nominal characteristics, as well as the parameters that can be analyzed may vary.

As suggested in RFC 3272[1] our LSP level monitoring performs end-to-end monitoring of individual LSP statistics. In addition to the actual bandwidth utilization, we also maintain $P H Y$ status, loss, signal quality, delay and activity statistics which can indicate wireless link stability with a varying significance depending on the QoS requirements of the payload. This receiving side monitoring measures the actual end-to-end characteristics of an LSP and is therefore mandatory to verify that an LSP receives the agreed end-to-end QoS handling. The per-LSP QoS requirements are installed at each node on the path during the LSP setup procedure together with the LSP forwarding state. This monitoring approach can be implemented by passive and feedback-free analysis of received datagrams which makes it therefore also suitable for UDLs.

\subsection{Link Layer Message Forwarding}

Similar to IEEE 802.11s, forwarding mechanisms for control traffic are implemented at the data link layer. User traffic is forwarded exclusively via LSPs and is therefore not affected by this design decision. Since all control communication takes place among MIMF entities, but with different forwarding requirements, we provide multiple generic message forwarding schemes to the NET_SAP which can than utilize the most appropriate one for each CG-WMAN control communication. The following messaging schemes are being provided:

- Management LSP

- Controlled flooding towards the destination, mainly the management entity

- Explicit source routing

Messaging via the management LSP is the preferred mechanism and is commonly relied upon unless the LSP has not yet been established or is down due to a link failure or network partitioning. 
The most basic hop-by-hop data link layer forwarding scheme is implemented via a controlled broadcast flooding towards the destination node. In the centralized approach, this destination node is in most cases the management entity, which by itself periodically floods mesh info messages into the mesh network. Using this information, the controlled flooding scheme can therefore direct the flooding towards the management entity. The controlled flooding approach is related to distance vector routing as it is used by many WMN routing protocols such as Ad hoc On-Demand Distance Vector Routing Protocol (AODV) 14]. In the presence of UDLs, the flooding control mechanism is by-passed to ensure forwarding to the destination. This mechanism is not expected to perform highly efficient forwarding, it is rather a last resort if all other means to forward control messages fail.

If enough topology knowledge is available at the sending node, it may use an explicit source routing mechanisms which precisely describes the links to be traversed. Links are described via LinkIDs which consists of the EUI-64 address of the sending node's interface and the EUI-64 address of the receiving node's interface. The management entities which maintain the link state table of their area typically have all the information to calculate the source route to a destination node. It is, in fact, the same mechanism that is used to calculate paths for LSPs. Hence, the management entity can utilize explicit source routing themselves, or can provide other nodes with custom source routing information, for example, from node A to node D and back, see Figure 6. If UDLs are present, the forward and return route might not be symmetric, but it will be ensured that the return route will traverse each designated node visited by the forward route.

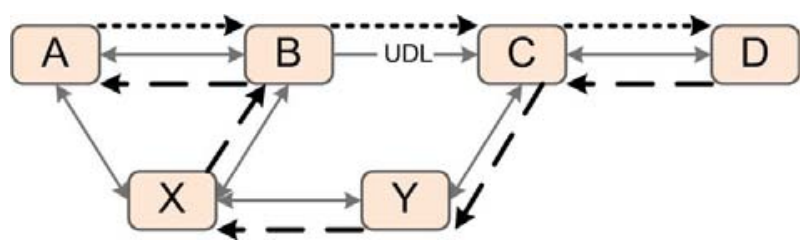

Fig. 6. The Explicit source routing scheme ensures that the same designated nodes are traversed

The explicit source routing mechanisms can operate in two different modes. In the default mode, the datagram is forwarded by intermediate nodes and only presented to the target module at the end of the route. In an alternative mode, the datagram is presented to the target module at each intermediate hop. This mode is, for example, used by the RSVP-TE-like path setup protocol, where state needs to be configured in each hop and is carried along the path.

\subsection{Path Management Protocol}

Our proposed CG-WMAN Path Management Protocol (PMP) is loosely based on the concepts of RSVP-TE and Path Computation Element Protocol (PCEP) 
[18] and is realized via MIMF messaging. PMP features a public interface which provides primitives for any CG-WMAN mesh node to

- request a path computation from a one or a set of PCEs

- set up an LSP and associated QoS and MAC layer state

- tear down an LSP and associated QoS and MAC layer state

PATH_REQUEST: To request the computation of a new path, this message is sent to the routing module specifying the source and destination node IDs of the path as well as the QoS requirements. Via the Response message, the routing module can return none, one or multiple possible paths to choose from. No resources are reserved at this point.

PATH_SETUP: The requesting node may then send a message to the routing function specifying the chosen path. The routing function will then try to allocate the resources and signal the setup of the associated LSP. Once completed, a Response message will be returned indicating the result of the setup procedure.

PATH_TEAR_DOWN: An established LSP may be torn down using this message. A Response message is returned when the procedure has completed indicating the result of the procedure.

Additionally, PMP provides a set of internal primitives which are used by the routing module to

- signal the setup of an LSP using explicit source routing

- signal the tear down of an LSP using explicit source routing

- configure FRR backup LSPs

- manage FRR event triggers

- retrieve LSP label and statistics for debugging purposes

When the routing function receives a PATH_SETUP message, it sends an explicitly source routed LSP setup message using the MIMF link layer forwarding service which is forwarded hop-by-hop along the path to be set up. Each node will assign a local outgoing label for this new LSP and store this label in the setup packet, so that it can be signaled to the next downstream node as incoming label. In the presence of UDLs, data link layer addresses of link local neighbors can not be learned. However, the LinkID consists exactly of this information, the EUI64 address of the outgoing interface and the EUI-64 address of the destination interface. A new LSP is identified at the ingress node using that nodes outgoing label.

A PATH_TEAR_DOWN message triggers a similar procedure to tear down an LSP identified by its ingress node's label.

For debugging purpose, a PATH_COLLECT_STATS message can be sent along the path of an LSP to collect the local labels associated with this LSP as well as related performance statistics.

FRR backup LSPs are signaled similarly to regular LSPs. The decision which LSP or segments thereof are to be protected with a backup is based on operator policies. After the backup LSP has been signaled, the nodes along the protected 
segments can be configured with multiple specific source routes towards the PLR. If no specific source routes have been configured, the controlled broadcast delivery mechanism is used. In the event of a link breakage, signaling might be impacted, as well. Hence, multiple paths and forwarding mechanisms could be used in parallel.

\section{Use Case: Multicast}

In the previous section we have presented our approach to seamlessly integrate UDLs into a CG-WMAN so that they can be utilized when beneficial for given payload characteristics or receiver distributions. We envisage a number of different use case where UDLs can increase the overall network efficiency, throughput or reliability.

UDLs based on, for example, DVB-T offer a very robust transport, a high spectral efficiency and are not impacted by any channel access protocol overhead. Hence, they are suitable for any kind of feedback-free content delivery from broadcast multimedia content to network topology updates. The latter one can benefit from the fact that a datagram is received at all nodes isochronously. Properly implemented, even network timing or synchronization tasks could be realized.

Single source 1-to-N multicast routing within a CG-WMAN with overlay cells can be configured in different ways depending on operator policies, receiver distribution and QoS requirements:

- In-mesh hop-by-hop multicast tree forwarding

- Single hop broadcast via an overlay cell

- Using a combination of the above
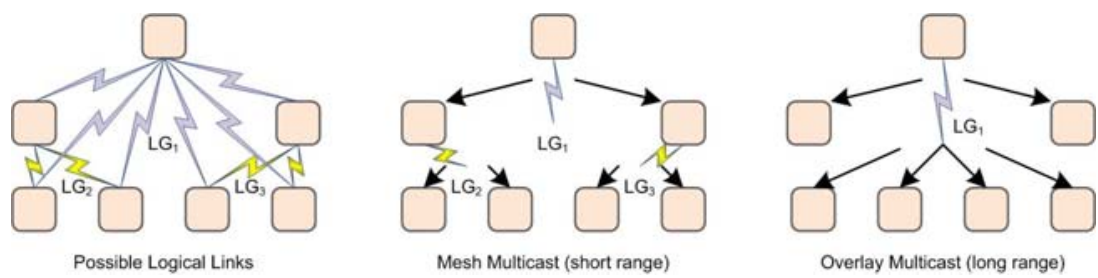

Fig. 7. Multicast routing can use in-mesh hop-by-hop forwarding or overlay cells

In order for the routing module to calculate the optimal multicast forwarding tree, a cost function is required which combines the information provided by the link group model, the link state table and the path database of established LSPs. From the link state table of logical links and the path database the topological receiving node distribution of a multicast tree can be determined. The result is a set of hop-by-hop forwarding trees, since in most cases multiple options 
will exist to form a tree covering all nodes, see Figure 7 Taking the link group information into account, the algorithm can determine which links belong to the same link group and if the nodes could be reached with a single link local broadcast transmission and which Modulation and Coding Scheme (MCS) must be used to reach the node with the weakest link conditions in the group. A lower MCS yields a lower spectral efficiency $(E)$. To calculate the costs of the resources to be allocated for an LSP segment in a link group, the number of nodes $(N)$ in this link group, the costs of its resources $(C)$, i.e. bandwidth, and the scheduling or channel access overhead $(O)$ are required. The latter varies heavily depending on the technology, its MAC layer design and the payload characteristics. For example, the IEEE 802.11 MAC is very inefficient when small (i.e. Voice-over-IP (VoIP)) datagrams are sent, since before sending each datagram the contention-based channel access procedure must be executed. This often requires more time than the actual datagram transmission.

Hence, the costs of LSP resources in a link group $C_{L S P}$ can be expressed as:

$$
C_{L S P}=\frac{C \cdot O}{E \cdot N}
$$

$C$ and $O$ are constant for a given LSP and its payload's characteristics. In the example depicted in Figure $8, E$ has a lower bound of $E_{\min }=0.5 \mathrm{bits} / \mathrm{s} / \mathrm{Hz}$ and an upper bound of $E_{\max }=4.0 \mathrm{bit} / \mathrm{s} / \mathrm{Hz}$ and may therefore vary depending on the receiving node distribution in the link group. Since $E$ is bound and $N$ may raise to $\infty, C_{L S P}$ decreases reciprocally proportional to the number of nodes, for $N>>\frac{E_{\max }}{E_{\min }}$. If $N$ is in the order of $\frac{E_{\max }}{E_{\min }}$, however, adding one distant node can significantly decrease $E$ and thus increase $C_{L S P}$.

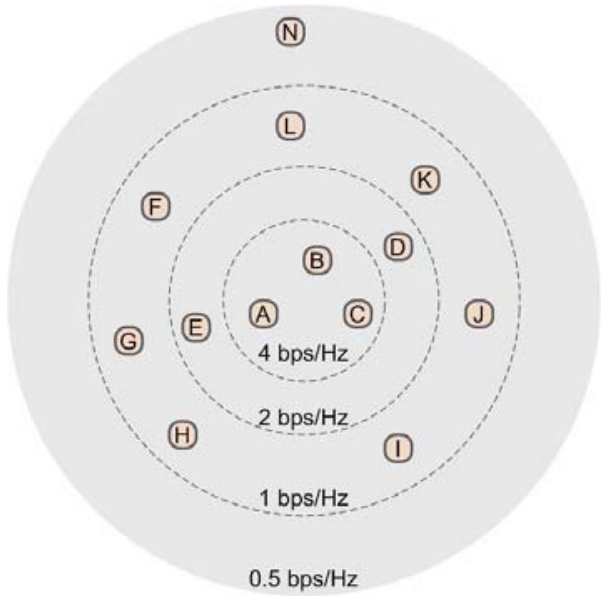

Fig. 8. The spectral efficiency decreases when a lower MCS is required to reach distant receivers or to increase reliability 
For example, in Figure 8 , if only node $\mathrm{A}$ is present, $E=E_{\max }$. If nodes $\mathrm{B}$ and $\mathrm{C}$ are added, $E$ remains constant and due the the increased number of nodes, the costs $C_{L S P}$ decrease. Let's assume, node N should be added. In this case $E$ would decrease significantly to $E_{\text {min }}$, while the number of nodes would just increase by one. It is now up to provider policies if node $\mathrm{N}$ would be allowed to join the link group (i.e. for premium customers), would be offered the payload via other links, or be denied access (i.e. for low-cost resale customers).

The above considerations need to be applied to each link group of the multicast tree. The total costs of a multicast tree $C_{\text {tree }}$ can then be expressed as the sum of the costs of the $n$ link groups traversed:

$$
C_{\text {tree }}=\sum_{i=1}^{n} C_{L S P_{i}}
$$

Here, we assume that the individual local link group costs consideration do not affect other links or link groups which are part of the multicast tree or even the mesh network as a whole. A simple multicast tree computation algorithm therefore has to consider two optimization criteria, meeting the end-to-end QoS requirements of the payload while minimizing the total costs $C_{\text {tree }}$ of the resulting multicast tree.

A more complex algorithm might be required to take into account dependencies between local link group optimization, provider policies, detailed payload characteristics and estimations of usage or receiver distribution pattern variations. The above approach could serve as a base line to quantify potential optimization gains of more complex algorithms.

Summarizing the above, we have shown that for large sets of nodes overlay cells offer a viable solution to keep multicast traffic off the regular mesh links. In cases of smaller sets of nodes, multiple trade-offs need to be considered to find the optimal multicast tree.

\section{Conclusion and Future Work}

We have presented our proposal to seamlessly integrate unidirectional technologies at the data link layer into a CG-WMAN. We have shown that given the constraints of a CG-WMAN, this is a suitable approach. In order to optimally utilize overlay cells, we have discussed which requirements routing algorithms need to fulfill and which trade-offs to consider.

Future work will look into autonomous adjustments of link group costs according to operator policies and a more advanced multicast group computation algorithm taking into account dependencies between local link group optimization. We will also evaluate additional use cases such as the (temporary) increase of downstream bandwidth or the use of overlay cells to transmit mesh network management messages.

The work described in this paper is a work in progress. At the time of writing, the described CG-WMAN is being implemented using our C++ Simple and Extensible Network Framework (SENF) 7] and its network emulator which allows 
for a mixed-mode validation of our design as well as an algorithm evaluation using real and emulated network interfaces. A multi-core Linux PC can emulate about 250 nodes, which allows us to evaluate, and optimize our proposed multicast routing algorithm.

\section{Acknowledgment}

The research leading to these results has received funding from the European Community's Seventh Framework Programme (FP7/2007-2013) under grant agreement $n^{\circ} 214994$. The views and conclusions contained here are those of the authors and should not be interpreted as necessarily representing the official policies or endorsements, either expressed or implied, of the CARMEN project or the European Commission.

\section{References}

[1] Awduche, D., Chiu, A., Elwalid, A., Widjaja, I., Xiao, X.: Overview and Principles of Internet Traffic Engineering. RFC 3272 (Informational). Updated by RFC 5462 (May 2002)

[2] Banchs, A., Bayer, N., Chieng, D., de la Oliva, A., Gloss, B., Kretschmer, M., Murphy, S., Natkaniec, M., Zdarsky, F.: Carmen: Delivering carrier grade services over wireless mesh networks. In: Proc. IEEE 19th International Symposium on Personal, Indoor and Mobile Radio Communications, PIMRC 2008, September 15-18, pp. 1-6 (2008)

[3] Deissner, J., Fettweis, G.P.: Increased capacity through hierarchical cellular structures with inter-layer reuse in an enhanced gsm radio network. Mob. Netw. Appl. 6(5), 471-480 (2001)

[4] Farrel, A., Vasseur, J.-P., Ash, J.: A Path Computation Element (PCE)-Based Architecture. RFC 4655, Informational (August 2006)

[5] Gundavelli, S., Leung, K., Devarapalli, V., Chowdhury, K., Patil, B.: Proxy Mobile IPv6. RFC 5213 (Proposed Standard) (August 2008)

[6] Haddad, E., Gregoire, J.-C.: Implementation issues for the deployment of a wmn with a hybrid fixed/cellular backhaul network in emergency situations. In: Proc. 1st International Conference on Wireless Communication, Vehicular Technology, Information Theory and Aerospace \& Electronic Systems Technology Wireless, VITAE 2009, pp. 525-529 (2009)

[7] http://senf.berlios.de (accessed 22-April-2009)

[8] Huang, Q., Ko, K.-T., Chan, S., Iversen, V.B.: Loss performance evaluation in heterogeneous hierarchical networks. In: Mobility 2008: Proceedings of the International Conference on Mobile Technology, Applications, and Systems, pp. 1-7. ACM, New York (2008)

[9] Kompella, K., Rekhter, Y.: IS-IS Extensions in Support of Generalized MultiProtocol Label Switching (GMPLS). RFC 5307 (Proposed Standard) (October 2008)

[10] Kretschmer, M., Ghinea, G.: Seamless integration of unidirectional broadcast links into qos-constrained broadband wireless mesh access networks. In: Proc. The 4th International Conference for Internet Technology and Secured Transactions (2009) 
[11] Liu, B., Thiran, P., Towsley, D.: Capacity of a wireless ad hoc network with infrastructure. In: MobiHoc 2007: Proceedings of the 8th ACM international symposium on Mobile ad hoc networking and computing, pp. 239-246. ACM, New York (2007)

[12] Moy, J.: OSPF Version 2. RFC 2328 (Standard) (April 1998)

[13] Pan, P., Swallow, G., Atlas, A.: Fast Reroute Extensions to RSVP-TE for LSP Tunnels. RFC 4090 (Proposed Standard) (May 2005)

[14] Perkins, C., Belding-Royer, E., Das, S.: Ad hoc On-Demand Distance Vector (AODV) Routing. RFC 3561 (Experimental) (July 2003)

[15] Raj, A., Ibe, O.C.: A survey of ip and multiprotocol label switching fast reroute schemes. Comput. Netw. 51(8), 1882-1907 (2007)

[16] Reaz, A., Ramamurthi, V., Ghosal, D., Benko, J., Li, W., Dixit, S., Mukherjee, B.: Enhancing multi-hop wireless mesh networks with a ring overlay. In: Proc. 5th IEEE Annual Communications Society Conference on Sensor, Mesh and Ad Hoc Communications and Networks Workshops SECON Workshops 2008, pp. 1-6 (2008)

[17] Rosen, E., Viswanathan, A., Callon, R.: Multiprotocol Label Switching Architecture. RFC 3031 (Proposed Standard) (January 2001)

[18] Vasseur, J., Roux, J.L.: Path Computation Element (PCE) Communication Protocol (PCEP). RFC 5440 (Proposed Standard) (March 2009)

[19] Yu, J.Y., Chong, P.H.J., Yang, M.: Performance of microcell/macrocell cellular systems with reuse partitioning. In: Mobility 2006: Proceedings of the 3rd international conference on Mobile technology, applications \&amp; systems, p. 51. ACM, New York (2006)

[20] Zhou, P., Manoj, B.S., Rao, R.: A gateway placement algorithm in wireless mesh networks. In: WICON 2007: Proceedings of the 3rd international conference on Wireless internet, Brussels, Belgium, pp. 1-9. ICST (Institute for Computer Sciences, Social-Informatics and Telecommunications Engineering) (2007) 